\title{
Mathematical modeling of wave processes and transport of bottom materials in coastal water areas taking into account coastal structures
}

\author{
Sofiya Protsenko ${ }^{1, *}$, Tatyana Sukhinova ${ }^{2}$ \\ ${ }^{1}$ Don State Technical University, Rostov, Russia \\ ${ }^{2}$ Southern Scientific Center of the Russian Academy of Sciences, Rostov, Russia
}

\begin{abstract}
The necessity of numerical modeling of the mechanisms of formation of the field of suspension and sediment flow determines the urgency of the problem under study. The process of developing shallow coastal zones, with constructive transformation and changes in coastal reliefs, is becoming increasingly important. Assessment of the hydrodynamic impact on shore protection structures and coastal structures installed at the bottom of a shallow water body is an important task at present. With a constructive transformation of the reliefs, one should take into account the dynamics of the formation of the coast investigate the formation of the profile of the bottom in the coastal zone of the reservoir under the influence of wave processes. For determining the dynamics and trend of phenomena occurring in the coastal waters and predicting possible interventions in the ecosystem, the need to build mathematical models of the transport processes of matter in the coastal waters, taking into account the presence of coastal structures, is actualized.
\end{abstract}

\section{Introduction}

There is a significant number of studies of dynamic processes occurring in the coastal zone of water bodies at present. Laboratory experiments performed by Russian and foreign scientists have revealed many regularities of formation of fields of a suspension under the influence of currents. The quantitative estimation and prediction of the distribution of suspended matter can be carried out using mathematical modeling, taking into account laboratory data obtained earlier.

Despite the large number of experimental and theoretical works aimed at the study of the specific dynamic processes of the coastal zone, the effectiveness of the proposed approaches are far from practical necessity. The difficulties of the research include the narrow scope of the models constructed on the basis of laboratory studies, the complexity of conducting experiments in a real sea, and, as a result, data limitations comprehensive full-scale measurements. Obtaining analytical solutions due to the nonlinearity and multidimensionality of the equations of NavierStokes equations underlying the considered problems, possible only in a very limited number of cases.

One of the most effective methods of investigation of real processes of hydrodynamics at the present time is the numerical simulation. For mathematical modeling of hydrodynamic processes in the reservoirs is an urgent problem of construction and practical use of computationally-efficient methods, the use of which would allow to obtain sufficiently accurate approximate numerical solution. Mathematical modeling of natural systems, including shallow ponds, in addition to, and in many cases allows to exclude expensive full-scale experiments with a real ecosystem. The use of mathematical models allows to predict the possible effects of changes in bottom topography due to natural climatic conditions or human intervention in the ecosystem of the pond. The development of information technology allows to significantly increase the scope and increase the complexity of the modeled task to expand the circle of users.

Wave processes are the main source of energy in the coastal zone of the reservoir, which significantly influences its dynamics; due to the fact that a successful field experiments with natural systems is difficult, and laboratory simulations, has a narrow specialization, mathematical modeling becomes an important tool for the analysis and management of coastal water systems; for the prediction of dynamic processes of the coastal zone there is a need of mathematical modeling of processes of movement of sediment in areas characterized by where the water is shallow under the influence of surface gravity waves.

There is a need to build models of three-dimensional wave processes and sediment transport, adapted for the simulation of hydrodynamic wave processes in the presence of various coastal structures, the scope of which is to build the velocity field and pressure of water environment, assessment of hydrodynamic impacts on coast protection works and coastal structures in the presence of surface waves, placed on the bottom of a shallow pond.

\footnotetext{
* Corresponding author: rab55555@rambler.ru
} 
In accordance with the intended purpose, it is necessary to solve following tasks: to justify the importance of solving problems, to carry out a review of existing approaches to the problem; to analyze existing models of sediment transport in aquatic ecosystems, and to analyse the approaches to mathematical modeling of these processes in aquatic ecosystems, classifying describing their mathematical models; to develop a model of sediment transport in shallow waters, allowing to predict the dynamics of the bottom relief; to develop a set of interrelated models, three-dimensional wave processes and sediment transport, adapted for hydrodynamic modelling of wave processes in the presence of various shoreline structures and to assess the hydrodynamic impact on coast protection works and coastal structures in the presence of surface waves.

\section{Mathematical model of sediment transport}

To describe the dynamics of marine sediments, the equations that describe the reformation of the coastal zone of water bodies are applied in the work, where water and solid particles move in one direction. The equations of the process of sediment transport [1-5] can be written in the following form:

$$
\begin{gathered}
(1-\varepsilon) \frac{\partial H}{\partial t}+\frac{\partial Q_{x}}{\partial x}+\frac{\partial Q_{y}}{\partial y}=0 \\
\vec{Q}=\left\{\begin{array}{l}
A \varpi d|\vec{\psi}|^{\beta-1} \vec{\psi}, \quad|\tau| \geq \tau_{b c}, \vec{\psi}=\frac{\vec{\tau}}{\left(\rho_{1}-\rho_{0}\right) g d}, \\
0, \quad|\tau|<\tau_{b c} ;
\end{array}\right.
\end{gathered}
$$

where $H$ - the depth of the bottom, measured from the unperturbed surface of the reservoir; $\varepsilon$ - the porosity of the soil; $\vec{Q}=\left\{Q_{x}, Q_{y}\right\}$ - the sediment load, $|\vec{Q}|=Q ; x, y$ - the horizontal Cartesian coordinates; $\tau_{b}$ - the tangential stress at the bottom; $\tau_{b c}$ - the critical value of the tangential stress at which the sediment transfer starts, $g$ - acceleration of gravity, $\rho$ - the fluid density, $A$ and $\beta$-dimensionless constants $(A=19.5, \beta=3)$, $\varpi-$ the wave frequency, $d-$ the characteristic of precipitation.

The shear stress for an inclined bottom surface is

$$
\vec{\tau}=\vec{\tau}_{b}-\alpha \sin S \vec{n},
$$

where $S(x, y, t)$ - the acute angle between the vector of the normal to the surface of the bottom and the vector of the gravitational force at time $t, \vec{n}-$ the unit vector directed towards the depth gradient, $\alpha \sin S-$ the additional tangential stress at the bottom of the reservoir, caused by gravitational forces. In case of $\left|\vec{\tau}_{b}\right|=0$, $|\vec{\tau}|=\tau_{b c}$ we have the equality: $\tau_{b c}=\alpha \sin \varphi_{0}, \varphi_{0}-$ the angle of the natural slope of the soil in the water.

Thus, the system of equations for the Schilds parameter takes the following form:

$$
\vec{\psi}_{S}=\frac{\vec{\tau}_{b}-\sin S \tau_{b c} \vec{n} / \sin \varphi_{0}}{\left(\rho_{1}-\rho_{0}\right) g d}, \vec{n} \operatorname{tg} S=\operatorname{grad} H,
$$

where $\psi_{S}$-Childs' parameter for the sloping bottom.

Taking into account the restrictions on tangential stresses at the bottom of the computational domain, the system of equations (1)-(3) takes the form [3-5]:

$$
\begin{gathered}
(1-\varepsilon) \frac{\partial H}{\partial t}+\operatorname{div}\left(k \vec{\tau}_{b}\right)= \\
=\operatorname{div}\left(k\left(\tau_{b c} / \sin \varphi_{0}\right) \operatorname{grad} H\right), \\
k=\frac{A \varpi d}{\left(\left(\rho_{1}-\rho_{0}\right) g d\right)^{\beta}}\left|\vec{\tau}_{b}-\frac{\tau_{b c}}{\sin \varphi_{0}} \operatorname{grad} H\right|^{\beta-1} . \\
\cdot h\left(\left|\vec{\tau}_{b}-\frac{\tau_{b c}}{\sin \varphi_{0}} \operatorname{gradH}\right|-\tau_{b c}\right),
\end{gathered}
$$

where $h(x)=\left\{\begin{array}{l}1, x \geq 0 \\ 0, x<0\end{array}\right.$ - the Heaviside's function.

The equation (4) is supplemented by the initial condition:

$$
H(x, y, 0)=H_{0}(x, y)
$$

At the boundary of the calculated region, there is no flow caused by the influence of gravitational forces:

$$
H_{0}^{\prime}(x, y)=0
$$

The information about the field of the velocity vector at the bottom of the reservoir is necessary to calculate the tangential stresses in the model of sediment transport. The system of shallow water equations is used to describe the movement of water and environment [69].

On the basis of the constructed algorithms were developed software intended for modeling of sediment transport in shallow waters [2-3]. The developed software, used to calculate two-dimensional velocity vector of the flow of the water environment, takes into account the turbulent exchange, the geometry of the seabed, wind currents and friction on the bottom.

Fig. 1-2 shows the results of numerical experiments modeling the dynamics of changes in bottom topography. The size of the grid is $200 \times 200$, step of spatial variable $0.1 \mathrm{~m}$, time step $0.01 \mathrm{~s}$, speed wind $5 \mathrm{~m} / \mathrm{s}$ and directed from left to right. Fig. 1 shows the initial bottom topography (bottom) and the position of the free surface (top).

The simulation of sediment transport has shown in about 20 minutes later, the geometry of the bottom begins to take on a conical structure. The velocity of the fluid flow the maximum in the peak part of the region (at minimum depths). A function of the elevation of the level takes positive values in the left part of the computational region (the windward side) and negative on the right. After 40 minutes there is the wavy structure of the function elevate the level not only in the left part, 
but in the region of peak depth. After 60 minutes enhanced oscillation functions elevate the level of the left part and in the region of the peak depth. The ridges are formed at the bottom of the computational region.

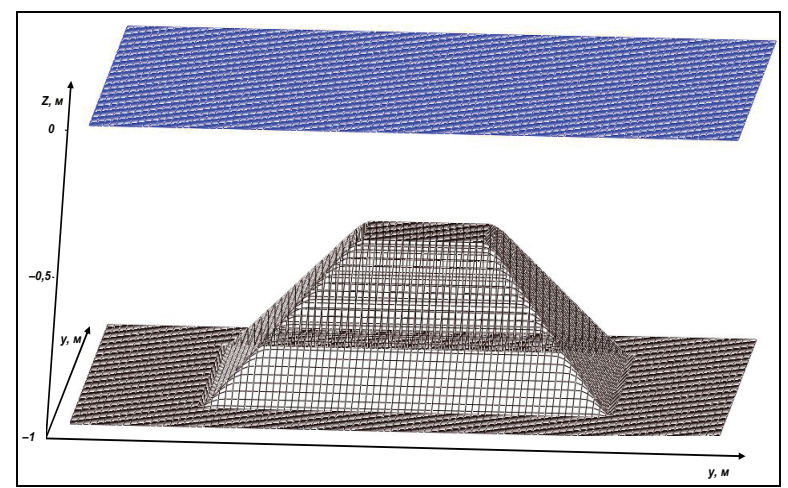

Fig. 1. The initial bottom topography and the position of the free surface

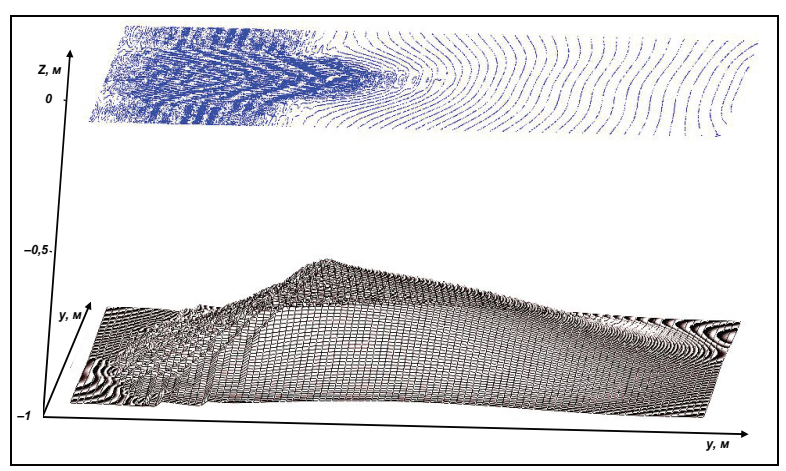

Fig. 2. The geometry of the bottom and the function of the elevation of the level after 80 minutes

After 80 minutes region with peak values of depth continues to shift to the left, there is an expansion region in the direction perpendicular to the movement of the wind (Fig. 2).

After 100 minutes region with peak values of depth continues to shift to the left, is the expansion of the area to the sides perpendicular to the motion of the wind, a function of the elevation of the level becomes highly oscillatory structure on the windward side, as well as the expansion of the area to the side. The results of the experiment allow us to analyse the dynamics of changes in the geometry of the bottom, the function of the elevation of the level of education wave patterns and sediment transport. This mathematical model and developed software allow to predict dynamics of change of the bottom topography, the appearance of a sea of ridges, their growth and transformation.

\section{Mathematical model of wave hydrodynamics}

For the mathematical description of the problem of wave dynamics of the fluid source equations are $[1,2]$ :

- the Navier-Stokes equation

$$
\begin{aligned}
& u_{t}^{\prime}+u u_{x}^{\prime}+v u_{y}^{\prime}=-\frac{P_{x}^{\prime}}{\rho}+\left(\mu u_{x}^{\prime}\right)_{x}^{\prime}+\left(\eta u_{y}^{\prime}\right)_{y}^{\prime}, \\
& v_{t}^{\prime}+u v_{x}^{\prime}+v v_{y}^{\prime}=-\frac{P_{y}^{\prime}}{\rho}+\left(\mu v_{x}^{\prime}\right)_{x}^{\prime}+\left(\eta v_{y}^{\prime}\right)_{y}^{\prime}+g
\end{aligned}
$$

- the continuity equation for incompressible fluid:

$$
u_{x}^{\prime}+v_{y}^{\prime}=0 .
$$

Equations (8)-(10) are considered with the following boundary conditions:

- at the bottom of the region:

$$
\begin{gathered}
P_{n}^{\prime}(x, y, t)=0, V_{n}(x, y, t)=0, \\
\rho \eta u_{y}^{\prime}(x, y, t)=-\tau_{x}(t), \\
\rho \mu v_{x}^{\prime}(x, y, t)=-\tau_{y}(t),
\end{gathered}
$$

- on the surface of the liquid:

$$
\begin{gathered}
u_{n}^{\prime}(x, y, t)=0, \quad v(x, y, t)=P_{t}^{\prime} / g \rho, \\
P_{n}^{\prime}(x, y, t)=0 ;
\end{gathered}
$$

- on a side border:

$$
\begin{gathered}
u_{n}^{\prime}(x, y, t)=0, \quad v_{n}^{\prime}(x, y, t)=0, \\
P_{n}^{\prime}(x, y, t)=\frac{\rho \Pi}{\tau L} ;
\end{gathered}
$$

where $V=(u, v)$ - the velocity vector of the aquatic environment; $P$ - the pressure; $\mu, \eta$ - the turbulent exchange coefficients in the horizontal and vertical directions, respectively; $g$ - acceleration of gravity; $\rho-$ the fluid density; $\tau_{x}, \tau_{y}-$ the components of the tangential stress at the bottom of the liquid; $\Pi-$ the flux of the velocity vector through the lateral surface; $L-$ the distance from the surface of the liquid to the bottom (the depth of the liquid taking into account the elevation of the level) at the lateral boundary. The axis $O x$ is combined with the surface of the unperturbed liquid and is directed toward the shore, the axis $O y$ - vertically down.

The tangential stress caused by bottom friction, according to the Van-Dorn law, is calculated by the formulas:

$$
\tau_{x}=\rho C_{p}(|V|) u|V|, \quad \tau_{y}=\rho C_{p}(|V|) v|V|,
$$

where $\quad C_{p}(|V|)=\left\{\begin{array}{l}0.0088,|V|<6,6 \mu / c, \\ 0.0026,|V| \geq 6,6 \mu / c\end{array} \quad\right.$ the dimensionless coefficient.

The calculated area is inscribed in a rectangle. For numerical realization of the discrete mathematical model 
of the formulated wave hydrodynamics problem, a uniform grid is introduced:

$$
\begin{gathered}
w_{h}=\left\{t^{n}=n \tau, x_{i}=i h_{x}, y_{j}=j h_{y} ;\right. \\
n=\overline{0, N_{t}}, i=\overline{0, N_{x}}, j=\overline{0, N_{y}} ; \\
\left.N_{t} \tau=T, N_{x} h_{x}=l_{x}, N_{y} h_{y}=l_{y}\right\},
\end{gathered}
$$

where $\tau$ - time step, $h_{x}, h_{y}$ - steps to space, $N_{t}-$ the upper bound on the time, $N_{x}, N_{y}$-borders in space.

The calculated cells are rectangles, they can be filled, partially filled, or empty. The cell centers and nodes are $h_{x} / 2$ and $h_{y} / 2$ by coordinates $x$ and $y$ respectively. We denote by $o_{i, j}$ "fullness» of the cell $(i, j)$. The degree of cell fullness is determined by the pressure of the liquid column inside this cell. If the average pressure at the nodes that belong to the vertices of the cell in question is greater than the pressure of the liquid column inside the cell, then the cell is considered to be full $\left(o_{i, j}=1\right)$. In this case, the "fullness» of the cell can be calculated by the following formula::

$$
o_{i, j}=\frac{P_{i, j} h\left(P_{i, j}\right)+P_{i-1, j} h\left(P_{i-1, j}\right)}{\rho g h_{y}},
$$

The results of numerical experiments for calculating the movement of the aquatic environment in the coastal water area are shown in Fig. 3, which shows the change in the profile of the wave coming ashore in a different period of time, while the program displays the vectors of the velocity field, the palette shows the pressure field.

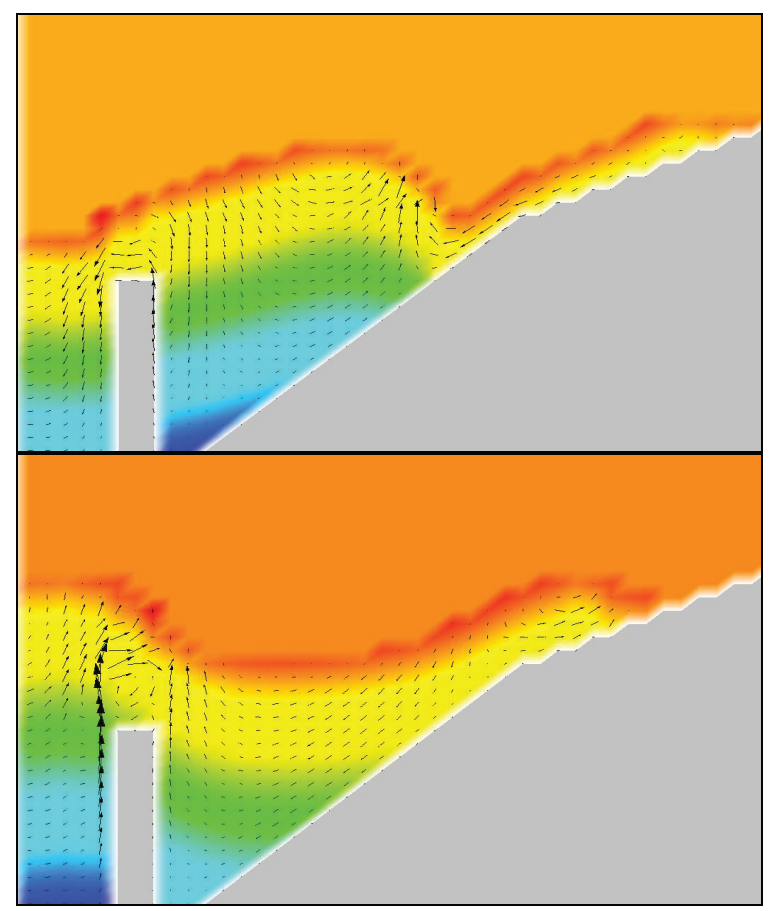

Fig. 3. The dynamics of the change in the profile of the elevation level of a liquid in the case of an artificial obstacle (breakwater)

\section{Mathematical model of wave processes, taking into account the presence of above-water coastal structures}

A three-dimensional hydrostatic model was used to construct a two-dimensional mathematical model of the motion of an aquatic environment. The initial equations of hydrodynamics are:

- Navier-Stokes euations system

$$
\begin{aligned}
& u_{t}^{\prime}+u u_{x}^{\prime}+v u_{y}^{\prime}+w u_{z}^{\prime}=-\frac{1}{\rho} P_{x}^{\prime}+\left(\mu u_{x}^{\prime}\right)_{x}^{\prime}+\left(\mu u_{y}^{\prime}\right)_{y}^{\prime}+\left(\eta u_{z}^{\prime}\right)_{z}^{\prime}, \\
& v_{t}^{\prime}+u v_{x}^{\prime}+v v_{y}^{\prime}+w v_{z}^{\prime}=-\frac{1}{\rho} P_{y}^{\prime}+\left(\mu v_{x}^{\prime}\right)_{x}^{\prime}+\left(\mu v_{y}^{\prime}\right)_{y}^{\prime}+\left(\eta v_{z}^{\prime}\right)_{z}^{\prime},(16)
\end{aligned}
$$

- the continuity equation for incompressible fluid

$$
u_{x}^{\prime}+v_{y}^{\prime}+w_{z}^{\prime}=0
$$

- the equation of hydrostatics

$$
P=\rho g(z+\xi) \text {. }
$$

The system of equations (16)-(18) is considered with the following boundary conditions: at the bottom the condition of impenetrability and friction $\rho_{v} \eta u_{n}^{\prime}=\tau_{x, b}(t)$, $\rho_{v} \eta v_{n}^{\prime}=\tau_{y, b}(t), V_{n}=0$, on the surface sets level rise and wind stress $\quad \rho \eta u_{n}^{\prime}=-\tau_{x, p}(t), \quad \rho \eta v_{n}^{\prime}=-\tau_{y, p}(t)$, $w=-\xi_{t}^{\prime}$, on the lateral boundaries, the condition of sliding without friction $u_{n}^{\prime}=0, v_{n}^{\prime}=0, \xi_{n}^{\prime}=0$, where $\xi$ - the raise function level (function of elevation), $V=\{u, v, w\}$ - the velocity vector of the aquatic environment, $P$ - the pressure, $\mu, \eta-$ the turbulent exchange coefficients in the horizontal and vertical directions, respectively, $g$ - the acceleration of gravity, $\rho$ - the density of the fluid, $\tau_{x}, \tau_{y}$ - the tangential stress at the bottom of the fluid, axis $O x, O y$ have a horizontal direction.

The equation calculate the pressure in hydrostatic approximation in the presence of surface design, installed on the bottom of the reservoir [14]:

$$
P=\rho g(z+\chi)+P_{g}=\rho g\left(z+\chi+P_{g} / \rho g\right) .
$$

The function of elevation in the case of a surface body on the surface is $\xi=\chi+P_{g} / \rho g$, where $\chi-$ the function describing the geometry of the bottom of the surface body.

The constructed program complex allows to specify the shape and intensity of the source of oscillations, as well as the geometry of the above-water object. The results of numerical experiments on the simulation of the propagation of wave hydrodynamic processes in the flow of an aqueous medium around a surface body, taking into account the geometries of the bottom of the object 
located in the liquid and the bottom of the reservoir, are shown in Fig. 5.

As an example of the practical use of a problemoriented program complex, the problem of calculating the hydrodynamic effect of support waves on structures is solved. Dimensions of the above-water structure: width $5 \mathrm{~m}$, length $10 \mathrm{~m}$, immersion depth $20 \mathrm{~cm}$. The structure is installed on the bottom of the reservoir with 6 supports. The selected modeling site has dimensions of 50 by $50 \mathrm{~m}$ and a depth of $1 \mathrm{~m}$. The disturbance source is set at some distance from the above-water object.

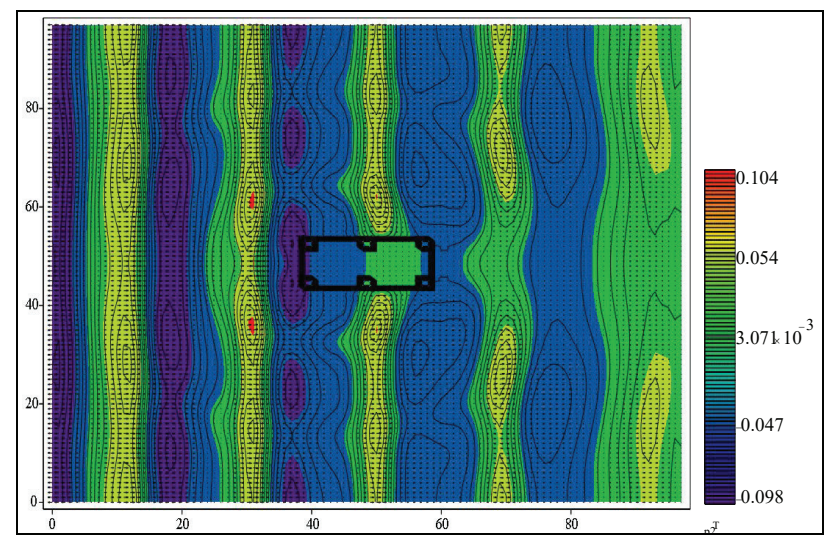

Fig. 4. Movement of the aquatic environment

At the initial time, the liquid is at rest. It is required to determine the subsequent movement of the aquatic environment in the presence of a surface object on the surface and the hydrodynamic forces on the supports of the structure. To solve the problem, a grid of 100x100 dimensions was used, the time step is 0,01 seconds.

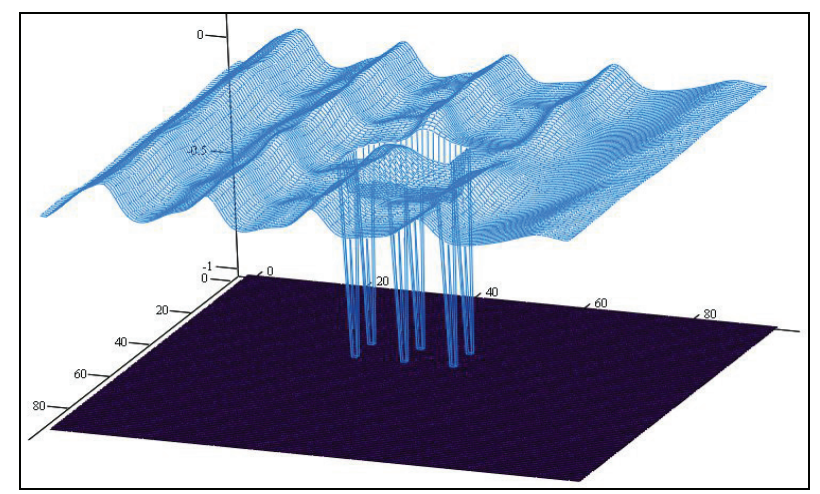

Fig. 5. The function of the elevation of the level when flowing over the water environment of a surface body having a support

Fig. 4, 5 shows that during the propagation of a plane wave that meets an obstacle in the form of a surface of the body, the reflection of wave oscillations from a fixed object, which in turn leads to a change of the wave profile. The source of vibrations distributed along the left border and has a sinusoidal form.

The results of numerical experiments on modeling the distribution of hydrodynamic wave processes and provide an opportunity to assess the impact of waves on structures having a support on the bottom of the pond. Fig. 6 shows the hydrodynamic force effects on the support surface of the structure installed on the bottom of the reservoir (Fig. 5, 6). The y-axis indicates the power loads in tons, the abscissa marks the time measured from the beginning of the oscillations in seconds.

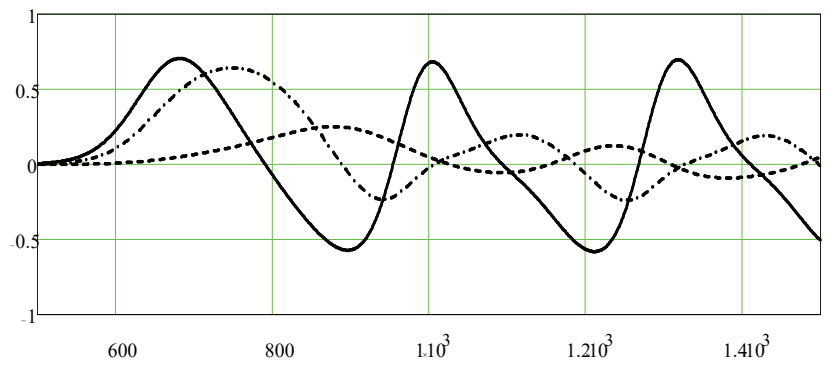

Fig. 6. Power loads on the supports of the above-water structure: 1 - on the front pair from the incoming wave, 2 - on the central pair, 3 - on the distant pair of supports

\section{Conclusion}

Models of sediment transport in coastal water systems are used in works related to protection from port and canal entry, construction of hydraulic structures, predictions of power impacts on coastal structures and bank protection structures.

The novelty of the scientific idea consists in the use of models of wave processes and transport of bottom materials in the coastal waters, taking into account the availability of coastal infrastructure facilities for studying hydrophysical processes in coastal water systems, as well as for constructing the velocity and pressure field of the aquatic environment, and assessing the hydrodynamic effect on shore protective structures and coastal structures in the presence of surface waves, installed on the bottom of a shallow water body.

Application of the research results is related to the planning of rational nature management: the construction of facilities and the use of specific sections of the shoreline, predicting possible reformation of the bottom.

The work was carried out with the financial support of the Program of Fundamental Research of the Presidium of the Russian Academy of Sciences I.33P, project No. 0256-20150078 .

\section{References}

1. E. Alekseenko, B. Roux, A. Sukhinov, R. Kotarba, D. Fougere, Computers and Fluids, 77, 24-35, (2013)

2. E. Alekseenko, B. Roux, A. Sukhinov, R. Kotarba, D. Fougere, Nonlinear Processes in Geophysics, 20 (2), 189-191 (2013)

3. N. Buzalo, P. Ermachenko, T. Bock, A. Bulgakov, A. Chistyakov, A. Sukhinov, E. Zhmenya, N. Zakharchenko, Procedia Engineering, 85, 84-93 (2014)

4. I. O. Leontev, Pribrezhnaya dinamika: volny, techeniya, potok osadkov, (GEOS, Moscow, 2001) [in Russian] 
5. A.V. Nikitina, A.I. Sukhinov, G.A. Ugolnitsky, A.B. Usov, A.E. Chistyakov, M.V. Puchkin, I.S. Semenov, Mathematical Models and Computer Simulations, 9 (1), 101-107 (2017)

6. E. A. Protsenko, Engineering journal of Don, 13, No. 3, 23-31 (2010) [in Russian]

7. E. A. Protsenko, News of southern Federal University. Technical Sciences, 97, No. 8, 71-75 (2009)

8. A.I. Sukhinov, A.E. Chistyakov, I.I. Levin, I.S. Semenov, A.V. Nikitina, A.A. Semenyakina, 5th International Conference on Informatics, Electronics and Vision, 1128- 1133 (2016)

9. A. I. Sukhinov, News of TRTU, № 3 (58), 228-235 (2006) [in Russian]

10. A. I. Sukhinov, Dvumernyye skhemy rasshchepleniya $i$ nekotoryye $i k h$ prilozheniya (MAKS Press, Moscow, 2005) [in Russian]

11. A. I. Sukhinov, A. E. Chistyakov, E. A. Protsenko, Izvestiya SFedU. Engineering Sciences, №8 (121), 32-44 (2011)

12. A.I. Sukhinov, A.E. Chistyakov, Mathematical Models and Computer Simulations, 4, 398-409 (2012)

13. A.I. Sukhinov, A.E. Chistyakov, A.V. Nikitina, A.A. Semenyakina, I. Korovin G. Schaefer, 2016 5th International Conference on Informatics, Electronics and Vision, 1134-1139 (2016)

14. A.I. Sukhinov, A.E. Chistyakov, A.A. Semenyakina, A.V. Nikitina, Computer researches and modeling, 8, No. 1, 151-168 (2016)

15. A.I. Sukhinov, A.E. Chistyakov, A.V. Shishenya, Mathematical Models and Computer Simulations, 6, No. 3, 324-331 (2014)

16. A.I. Sukhinov, A.E. Chistyakov, E.F. Timofeeva, A.V. Shishenya, Mathematical Models and Computer Simulations, 5, No. 2, 122-129 (2013)

17. A.I. Sukhinov, D.S. Khachunts, A.E. Chistyakov, Computational Mathematics and Mathematical Physics, 55, No. 7, 1216-1231 (2015).

18. A.I. Sukhinov, A.V. Shishenya, Mathematical Models and Computer Simulations, 5, N 3, 257-265 (2013)

19. V. S. Vasiliev, A. I. Sukhinov, Mathematical modelling, 15, No. 10, 17-34 (2003)

20.E.V. Yakushev, A.I. Sukhinov, Yu.F. Lukashev, S.Yu. Fomin, V.G. Yakubenko, Oceanology, 43, No 1, 39-47 (2003) 